\title{
Analysis of the propagation of terahertz surface plasmon polaritons on semiconductor groove gratings
}

\author{
M. Kuttge ${ }^{\text {a) }}$ and H. Kurz \\ Institute of Semiconductor Technology, RWTH-Aachen University, D-52056 Aachen, Germany \\ J. Gómez Rivas \\ FOM Institute for Atomic and Molecular Physics AMOLF, c/o Philips Research Laboratories, \\ High-Tech-Campus 4, 5656 AE Eindhoven, The Netherlands \\ J. A. Sánchez-Gil \\ Instituto de Estructura de la Materia, Consejo Superior de Investigaciones Científicas, Serrano 121, \\ E-28006 Madrid, Spain \\ P. Haring Bolívar \\ Institute of High Frequency and Quantum Electronics, University of Siegen, Hölderlinstrasse 3, \\ D-57068 Siegen, Germany
}

(Received 25 July 2006; accepted 7 November 2006; published online 23 January 2007)

We have investigated the propagation of terahertz (THz) surface plasmon polaritons (SPPs) on gratings formed by grooves structured in silicon wafers. These gratings exhibit a stop gap where SPPs are Bragg scattered. We observe a strong effect of the groove depth on the characteristics of the stop gap. To quantify the scattering strength of these structures we obtain the SPP attenuation length by measuring the transmission as a function of the number of grooves. We also determine the effective refractive index of the Bragg gratings using two different approaches: by measuring the transmittance through the gratings at different angles of incidence and from the phase of the transmitted $\mathrm{THz}$ pulses. The two approaches give results in good agreement. (C) 2007 American Institute of Physics. [DOI: 10.1063/1.2409895]

\section{INTRODUCTION}

Surface plasmon polaritons (SPPs) are electromagnetic waves coupled to the oscillation of free charge carriers at a conductor-dielectric interface, ${ }^{1}$ which have a field amplitude strongly confined to the surface. SPPs are thus very sensitive to the properties and structure of the surface, being widely used for spectroscopy of interfaces and sensing. ${ }^{2}$ The twodimensional character of SPPs makes it possible to confine electromagnetic radiation below the diffraction limit, offering unprecedented possibilities for subwavelength optics. ${ }^{3-7}$ A key aspect in surface plasmon optics or plasmonics is the control of the propagation of SPPs. A wealth of optical components for SPPs such as mirrors, beam splitters, couplers, and waveguides can be achieved by structuring the surface of the conductor. ${ }^{8-16}$ One possibility is a periodic modulation of the surface, which leads to Bragg scattering and the suppression of the transmission of SPPs in a range of frequencies known as the stop gap. Recently, the propagation of optical SPPs through gratings ${ }^{8-11}$ and two-dimensional photonic crystals ${ }^{12-14}$ has been experimentally investigated and simple plasmonic circuits have been demonstrated. ${ }^{17}$ At terahertz $(\mathrm{THz})$ and microwave frequencies, the absolute value of the permittivity of metals is very large, which leads to a weak field confinement of the SPPs to the surface and a poor scattering of surface plasmon at inhomogeneities. According to low order perturbation theories, ${ }^{18-20}$ the normalized gap width for Bragg gratings on metal surfaces has a small value

${ }^{\text {a)} E l e c t r o n i c ~ m a i l: ~ k u t t g e @ a m o l f . n l ~}$ at $\mathrm{THz}$ frequencies. Consequently, low frequency surface plasmon optics has been less thoroughly investigated than optical plasmonics. Semiconductors have a much lower permittivity than metals in the $\mathrm{THz}$ range, thus being more suitable for the excitation and confinement of low frequency SPPs modes to the surface ${ }^{21}$ and for low frequency surface plasmon optics. In Ref. 16 the optical studies on the propagation of SPPs through Bragg mirrors were extended to low frequencies by demonstrating the propagation of $\mathrm{THz}$ SPPs on semiconductors and the very efficient Bragg scattering with gratings of grooves structured on semiconductor surfaces.

In this article we explore the dependency of the $\mathrm{THz}$ stop gap on the groove depth, observing an asymmetric broadening and deepening of the gap for deeper grooves. We quantify the scattering strength of the gratings in terms of the SPPs attenuation length, by measuring the transmission of SPPs through gratings with different number of grooves. We also determine the effective refractive index of the grating for SPPs in two different ways: by measuring the conical transmittance of SPPs through the grating and from the phase of the transmitted SPPs pulses at normal incidence. The two methods give results which are in good agreement.

This manuscript is organized as follows: In Sec. II we describe the fabrication of the gratings and the experimental setup. The measurements of the propagation of SPPs through gratings with different groove depths are presented in Sec. III. In Sec. IV we show measurements of the scattering strength of the gratings by measuring the transmission through similar gratings with different number of grooves. 
The determination of the effective SPPs refractive index is presented in Sec. V. Section VI contains the conclusions.

\section{SAMPLES AND SETUP}

Gratings were structured on $n$ - and $p$-doped silicon wafers with doping concentrations of $N \approx 10^{18} \mathrm{~cm}^{-3}$ and $10^{19} \mathrm{~cm}^{-3}$, respectively. The real part of the permittivity of these materials at $0.3 \mathrm{THz}$ is $\varepsilon \approx-18$ for the $n$-doped $\mathrm{Si}$ and $\varepsilon \approx-52$ for the $p$-doped Si. These values of the permittivity were derived assuming validity of the Drude model for semiconductors in the $\mathrm{THz}$ frequency range. ${ }^{22}$ The gratings were structured by dicing parallel cuts with a programmable wafer saw. The metallic character, i.e., the negative real part of the permittivity of doped semiconductors at $\mathrm{THz}$ and microwave frequencies makes them suitable to sustain SPPs. Moreover, the much lower absolute value of their permittivity compared to that of metals at low frequencies results in a much shorter decay length for the SPPs perpendicular to the surface into air. ${ }^{1}$ The SPPs are therefore more strongly bound to the surface and show a higher sensitivity to the surface structure. A very efficient scattering of SPPs with inhomogeneities in an otherwise flat semiconductor surface is thus expected. ${ }^{23}$

For the measurements we used a $\mathrm{THz}$ time-domain spectrometer. ${ }^{24}$ For the generation and detection of $\mathrm{THz}$ radiation we used the femtosecond (fs) pulses of a Ti:sapphire laser which were split into two beams. One of these beams, the excitation beam, generated $\mathrm{THz}$ radiation on a surface field emitter, which consisted of a piece of InGaAs glued to a gold mirror. Due to the pulsed excitation of carriers by the fs-second laser the carrier density changes rapidly, generating a broadband $\mathrm{THz}$ pulse in the frequency range from 100 $\mathrm{GHz}$ up to $2.5 \mathrm{THz}$. The emitted $\mathrm{THz}$ radiation was collimated and focused onto an aperture to generate SPPs. ${ }^{25-28}$ The aperture was formed by the surface of the sample and a razor blade edge placed at a distance of $300 \mu \mathrm{m}$ above the surface. As reported in Ref. 25 the optimal height for the blades is on the order of the wavelength of the incoming radiation. In the $\mathrm{THz}$ frequency range this means a value of $h \approx 300 \mu \mathrm{m}$. Although aperture coupling has a poor efficiency, its major advantage is the broadband excitation of SPPs. The generated SPPs propagated $2 \mathrm{~cm}$ over the sample and were coupled back into free space electromagnetic radiation at a second aperture. The outcoupled radiation was detected by a photoconductive antenna, which was gated by the second beam of the fs laser. By varying the path length between the optical generation and detection beams, the amplitude of the THz field was detected in the time domain. The solid curve in Fig. 1(a) shows the THz field of a SPP pulse that has propagated over $2 \mathrm{~cm}$ on a flat surface. It is important to note that this technique allows the detection of both the amplitude and the phase of the $\mathrm{THz}$ pulses.

\section{STOP GAP DEPENDENCE ON THE DEPTH OF THE GROOVES}

When a SPP encounters an inhomogeneity at the surface, such as a groove, it is scattered. The strength of the scattering depends on the size of the inhomogeneity, i.e., the depth and width of the groove. If instead of one groove we have a

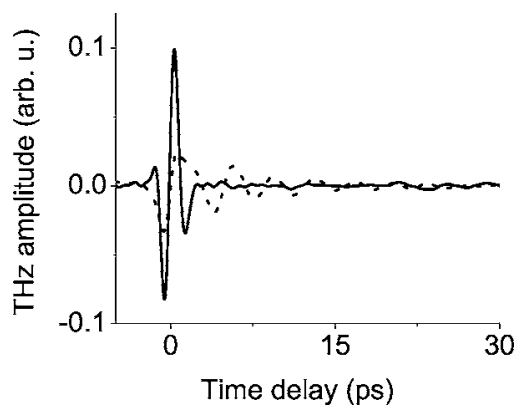

FIG. 1. (a) THz transients of SPPs propagating $2 \mathrm{~cm}$ on the surface of an $n$-doped silicon wafer. The solid curve corresponds to the propagation on a flat silicon surface and the dotted curve corresponds to SPPs on a Si surface that is structured with a grating. The grating consists of 30 grooves with lattice constant of $a=442 \mu \mathrm{m}$ and groove depth $d=100 \mu \mathrm{m}$.

periodic array of grooves or grating, interference of SPPs scattered at the different locations will lead to the formation of a stop gap or a frequency range where SPPs are Bragg reflected and the transmission through the grating vanishes. The dotted curve in Fig. 1(a) represents a THz transient for SPPs propagating over a grating of 30 grooves. The lower amplitude compared to the pulse on a flat surface (solid curve in the same figure), and the oscillatory component at longer time delays are the result of the scattering of SPPs by the grating.

To investigate the dependence of the stop gap with the depth of the grooves we used $p$-doped Si gratings. We measured the SPP transmission through gratings of 30 grooves with a grating period of $442 \mu \mathrm{m}$. The groove width was $221 \mu \mathrm{m}$. In Fig. 2(a) we show the transmittance, i.e., the power spectrum of the pulses transmitted through the gratings normalized by the spectrum of SPPs propagating on a flat surface, as a function of frequency for samples with different depth of the grooves: $30 \mu \mathrm{m}$ (open up-triangles), $90 \mu \mathrm{m}$ (circles), $150 \mu \mathrm{m}$ (open squares), and $220 \mu \mathrm{m}$ (down-triangles). For low frequencies the transmittance is close to one for all grove depths, as SPPs are not affected by the grating. The lower edge of the stop gap can be identified by the sharp decrease in the transmittance. At these frequencies, SPPs are Bragg scattered and the transmission through the grating is inhibited. The transmittance rises for higher

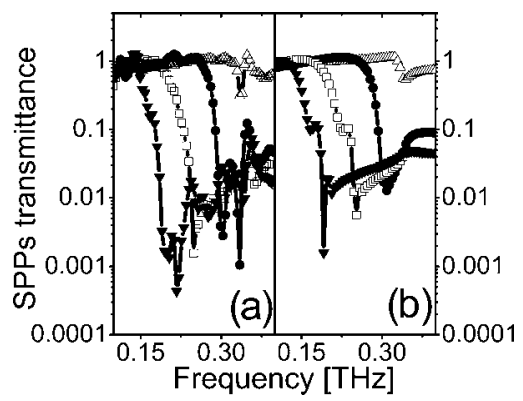

FIG. 2. Transmittance as a function of frequency of SPPs propagating through gratings of grooves with different depths structured on $p$-doped Si wafers. The curves in (a) are measurements for groove depths of $30 \mu \mathrm{m}$ (open up-triangles), $90 \mu \mathrm{m}$ (circles), $150 \mu \mathrm{m}$ (open squares), and $220 \mu \mathrm{m}$ (down-triangles). The transmittance calculated numerically for gratings with the same parameters, i.e., without any adjustable parameter, are shown in (b). 


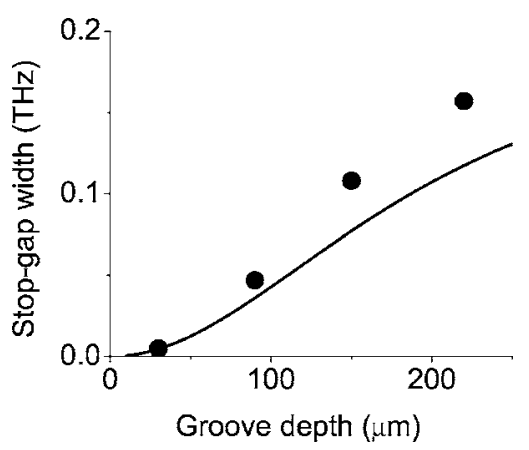

FIG. 3. Width of the SPPs stop gap as a function of the groove depth. The circles show the experimentally determined width while the solid curve represents a calculation.

frequencies but it does not reach one, which can be attributed to radiative losses due to outcoupling of SPPs by the grating (leaky SPPs). When we increased the groove depth the stop gap became deeper and it widened, leading to a shift of the band edge to lower frequencies. These effects can be understood by the increase of the scattering cross section of the individual grooves as they become larger. Although difficult to appreciate from the measurements, the broadening of the stop gap should be asymmetric, as first described by Barnes et al. in Ref. 20 for optical SPPs on metallic gratings. The energies of the modes at the band edges of the stop gap result from the electromagnetic field and charge distribution at the surface. As the groove depth is increased these distributions change, leading to a shift of the lower band edge to lower frequencies and of the upper edge to higher frequencies. However, the shift of the upper edge is limited by the frequency of electromagnetic radiation. SPPs with higher frequencies are coupled to free space electromagnetic radiation becoming leaky waves.

To validate our measurements we calculated numerically the SPP transmission through gratings with the same parameters of those in Fig. 2(a). The results of these calculations are plotted in Fig. 2(b). To calculate these curves the rigorous electromagnetic formulation of the Green's theorem surface integral equations has been employed. The experimental configuration is reproduced by a semi-infinite semiconductor surface on which the SPPs are excited by the use of a sinusoidal grating, the period of which is tuned to couple the beam incident at a given angle into a SPP. ${ }^{29}$ The field of the SPPs transmitted through the grating is obtained from the electromagnetic field intensity at the position of the outcoupling blade. Note that all the parameters in the calculations are known, and the good agreement with the experimental results is achieved without any fitting variable.

We estimated the width of the gap in the same approximation as in Refs. 18 and 19, namely, restricting ourselves to the first Bragg scattering of the SPP, but on the basis of the formalism of the reduced Rayleigh equation obtained through the use of an impedance boundary condition on a plane. $^{30}$ This width of the gap is represented in Fig. 3 with the solid curve. The sharp features on the experimental spectra and the radiative losses at high frequencies make it difficult to define the gap width from the experiments. To compare with the calculations we defined the lower band edge as

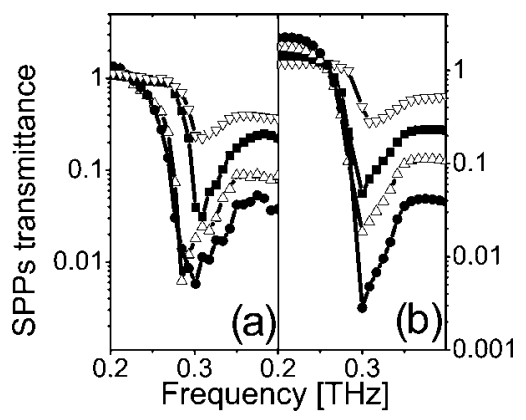

FIG. 4. Transmittance as a function of frequency of SPPs propagating through gratings of grooves with different numbers of grooves structured on $p$-doped wafers. The curves in (a) are measurements for 10 (open downtriangles), 20 (squares), 30 (open up-triangles), and 40 grooves (circles). The transmittance calculated numerically for gratings with the same parameters, i.e., without any adjustable parameter, are shown in (b).

the frequency at which the transmittance decreases to 0.1 , except for the grating with the shallowest groves where the transmittance decreases to less than 0.1 . For this sample the band edge was defined as the frequency of the lowest transmittance. The upper frequency edge was defined as the frequency at which radiative losses due to outcoupling by the grating become dominant, i.e., $0.35 \mathrm{THz}{ }^{16,31}$ The experimental width of the stop gap is represented in Fig. 3 by the circles. Recalling that no fitting parameters are used for the calculations and the complex experimental definition of the width of the gap, we found a remarkably good agreement between theory and measurements. This agreement deteriorated for deeper grooves, which can be attributed to the fact that the above mentioned impedance boundary condition underestimates the scattering strength of large grooves, leading to narrower gap widths.

\section{SCATTERING STRENGTH OF THE GRATINGS}

An important parameter for characterizing the gratings is the attenuation length $L_{\text {att }}$. The transmission of SPPs through a grating decreases exponentially with the number of grooves due to scattering. The attenuation length is the characteristic length of this exponential decrease, defining thus the scattering strength of the grating: A shorter $L_{\text {att }}$ means a stronger scattering. To obtain $L_{\text {att }}$ we measured the transmittance as function of the number of grooves keeping all the other parameters constant. In Fig. 4(a) we show the transmittance for 10 (open down-triangles), 20 (squares), 30 (open up-triangles), and 40 grooves (circles). The gratings were made of $p$-doped Si with a period of $442 \mu \mathrm{m}$, a groove width of $221 \mu \mathrm{m}$, and a groove depth $d=100 \pm 5 \mu \mathrm{m}$. As the number of grooves increased the transmittance at frequencies within the stop gap was reduced. The slightly different positions of the low frequency band edge are due to small variations in the depth of the grooves. We also observed that the transmittance at high frequencies decreased with the number of grooves as is expected from the increase of the radiative losses. If we compare the measurements to the numerical calculations shown in Fig. 4(b), we observe good agreement. The attenuation length can be determined by fitting the minimum transmittance at frequencies in the gap as a function of the number of grooves by a single exponential decay. In Fig. 


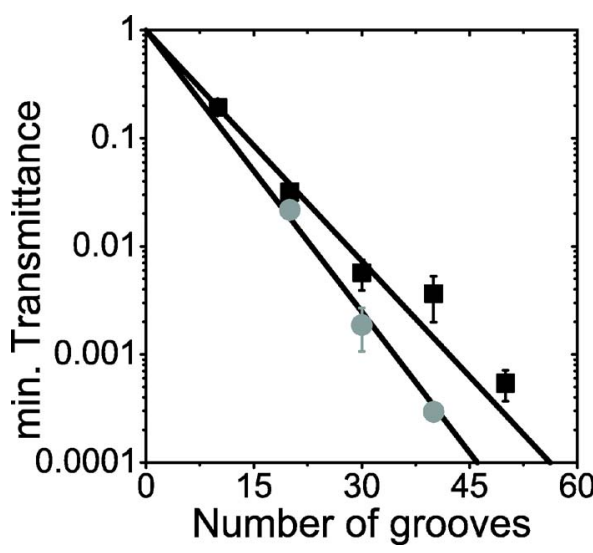

FIG. 5. Transmittance of SPPs through gratings of grooves at frequencies within the stop gap as a function of the number of grooves. The squares correspond to gratings made of $p$-doped silicon and the circles correspond to gratings of $n$-doped silicon. The lines are exponential fits to the measurements.

5 we have plotted this minimum transmittance for the gratings made of $p$-doped $\mathrm{Si}$ (squares) and of $n$-doped $\mathrm{Si}$ (circles). The lines in Fig. 5 are exponential fits to the data, from which we obtain $L_{\text {att }}=2.67 \pm 0.02 \mathrm{~mm}$ for $p$-doped $\mathrm{Si}$ and $L_{\text {att }}=2.30 \pm 0.03 \mathrm{~mm}$ for $n$-doped $\mathrm{Si}$. The shorter attenuation length for $n$-doped $\mathrm{Si}$ means a stronger and therefore more efficient scattering. ${ }^{16}$

The scattering of SPPs by a single groove is stronger for frequencies closer to the plasma frequency $\nu_{p}{ }^{32}$ At these frequencies the absolute value of the permittivity becomes small and SPPs are more strongly confined to the surface. The plasma frequency in semiconductors is determined by their doping concentration and the effective carrier mass. In our samples we have $\nu_{p}=7.4 \mathrm{THz}$ for $p$-doped $\mathrm{Si}$ and $\nu_{p}$ $=2.8 \mathrm{THz}$ for $n$-doped Si. For $n$-doped Si the plasma frequency is thus closer to the stop gap frequency, in accordance with the stronger SPP scattering observed for this material. We should mention that the plasma frequency of metals is much larger, typically a few hundred $\mathrm{THz}$, which leads to a less efficient scattering at low frequencies.

\section{EFFECTIVE REFRACTIVE INDEX}

The effective refractive index $n_{\text {eff }}$ describes the phase velocity of SPPs propagating on an inhomogeneous medium. We present here two methods to determine the SPPs effective refractive index of $\mathrm{THz}$ gratings. One method involves measuring the shift of the stop gap band edge for SPPs incident on the grating at different angles. The second method is a direct measurement of $n_{\text {eff }}$ from the phase information contained in the transmitted $\mathrm{THz}$ pulses.

In the case of normal incidence of SPPs on a grating, the wave vector of the SPPs $\boldsymbol{k}$ and the reciprocal lattice vector $\boldsymbol{G}$ of the grating are parallel. The Bragg condition is given by $k=n G / 2$, where $n$ is an integer, i.e., for normal incidence of SPPs on the gratings the wave vector has to be a multiple of half the reciprocal lattice vector. For non-normal incidence we have to consider the projection of the SPPs wave vector onto the reciprocal lattice vector. This projection is given by $k^{\prime}=k \cos \beta$, where $\beta$ is the angle formed by the SPPs wave vector and the reciprocal lattice vector of the grating. There-

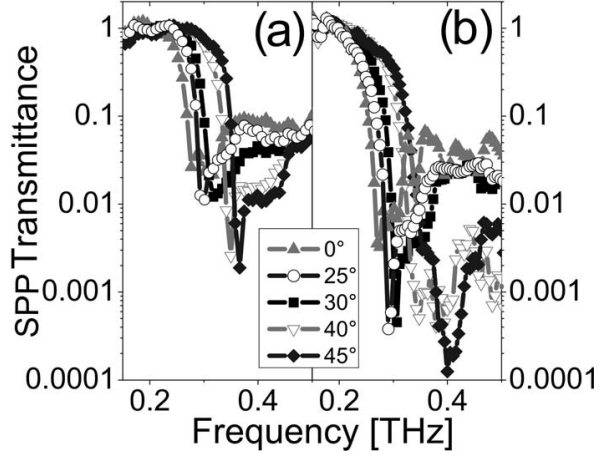

FIG. 6. Transmittance as a function of frequency for SPPs propagating on gratings of (a) $p$-doped and (b) $n$-doped $\mathrm{Si}$ for different angles of incidence. The gratings consist of 30 grooves with lattice constant $a=442 \mu \mathrm{m}$ and a groove depth $d=100 \mu \mathrm{m}$. The gratings are tilted an angle $\theta$ with respect to the direction of incidence of the SPPs.

fore, for conical (non-normal) propagation the modulus of the wave vector must be larger than for normal incidence to fulfill the Bragg condition, i.e., the band gap shifts to higher frequencies. For non-normal incidence, refraction of SPPs at the grating boundaries becomes important. If we assign to the whole grating an effective refractive index $n_{\text {eff }}$, SPPs incident at an angle $\theta$ on the grating are refracted. The SPPs propagate thus at a different angle $\beta$ inside the grating. This angle is given by $\sin \beta=1 / n_{\text {eff }} \sin \theta$. The position of the gap at an angle of incidence $\theta$ is thus given by

$$
\nu=\nu_{0} \frac{1}{\sqrt{1-\left(\sin \theta / n_{\mathrm{eff}}\right)^{2}}},
$$

where $\nu_{0}$ is the position of the gap for normal incidence. By measuring the gap for different angles of incidence we can thus determine the effective refractive index of the grating.

We have measured the propagation over a grating of $p$-doped Si turning the grating from $\theta=0^{\circ}$ up to $\theta=45^{\circ}$. The grating consisted of 30 grooves with a lattice constant $a$ $=442 \mu \mathrm{m}$ and a groove depth $d=99 \mu \mathrm{m}$. The transmittance as a function of frequency for a selected number of angles $\theta$ is shown in Fig. 6(a). Another series of measurements was done with a grating of $n$-doped $\mathrm{Si}$ with a groove depth of $d=97 \mu \mathrm{m}$. The other parameters were the same as for the $p$-doped grating. Figure 6(b) shows the transmittance of the $n$-doped sample as a function of the frequency for selected angles $\theta$. For both samples the gap shifts to higher frequencies for increasing angles as it was expected.

In Figs. 7(a) and 7(b) we show the spectral position of the gap $\nu$ extracted from the transmittance measurements of Figs. 6(a) and 6(b). To define $\nu$ we take the frequency at the low frequency band edge where the transmittance drops below a certain value. For $p$-doped $\mathrm{Si}$ the position is determined by 0.1 transmittance, while for $n$-doped $\mathrm{Si}$ by 0.01 transmittance. The solid curves in Fig. 7 are fits of Eq. (1) to the measurements. For the grating structured in $p$-doped $\mathrm{Si}$ we find an effective refractive index of $n_{\mathrm{eff}}$ $=1.05 \pm 0.01$ and a gap frequency for normal incidence $\nu_{0}$ $=(0.264 \pm 0.004) \mathrm{THz}$. The fit for the $\mathrm{n}$-doped $\mathrm{Si}$ grating yields $n_{\text {eff }}=1.09 \pm 0.01$ and $\nu_{0}=(0.261 \pm 0.004) \mathrm{THz}$. The values of $n_{\text {eff }}$ close to one are expected, because at low frequencies the dispersion relations of SPPs and free space electro- 
(a)

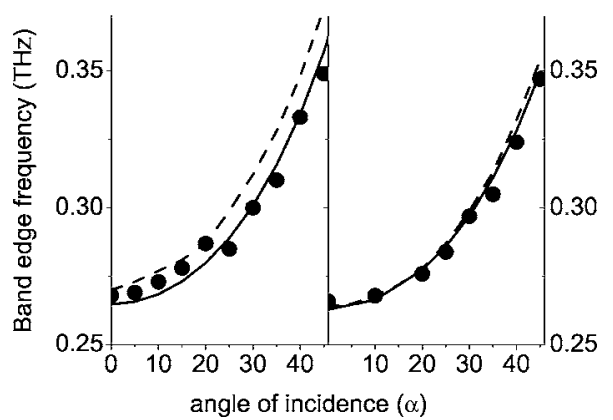

FIG. 7. Low-frequency band edge (circles) of the stop gap of SPPs as a function of the angle of incidence for a (a) $p$-doped and (b) $n$-doped $\mathrm{Si}$ grating of 30 grooves. The lattice constant is $a=442 \mu \mathrm{m}$ and the groove depth $d=100 \mu \mathrm{m}$. The solid curve is a fit of Eq. (1) to the data. The results of the numerical calculations (based on the approach mentioned in the text, for $d=120 \mu \mathrm{m}$ ) are shown as dashed curves.

magnetic radiation are similar. The effective refractive index of the grating structured in the $n$-doped wafer is larger than that of the $p$-doped one, consistent with the scattering strengths. Numerical results are also represented in Fig. 7 with the dashed curves. These results have been calculated on the basis of the theoretical formulation of the reduced Rayleigh equation, ${ }^{30}$ modified to incorporate oblique incidence. Good agreement is obtained with the experimental data.

The determination of the effective refractive index as described earlier is a time consuming procedure because several measurements at different angles are necessary. The effective refractive index can also be obtained from the phase of the transmitted pulses through the grating at normal incidence. Defining the phase difference $\Delta \varphi$ between the transmitted and the reference pulses, the dispersion relation $k(\nu)$ $=\Delta \varphi / L+2 \pi \nu / c$, where $L$ is the width of the structure, can be obtained. The analysis of the phase of our measurements gives us the phase difference $\Delta \varphi(\nu)$, and allows to calculate the dispersion relation $k(\nu)$. From the dispersion relation we can calculate the effective refractive index $n_{\text {eff }}$,

$$
n_{\mathrm{eff}}=k(\nu) \frac{c}{\omega} .
$$

We can now compare the values of the effective refractive index obtained by the two methods, i.e., the conical propagation and the phase analysis. In the analysis of the conical propagation measurements we have defined the position of the gap as the frequency at the low frequency band edge where the transmittance dropped below a certain value. These frequencies are $(0.264 \pm 0.004) \mathrm{THz}$ and $(0.261 \pm 0.004) \mathrm{THz}$ for the $p$ - and $n$-doped Si gratings, respectively. To compare the results we must take the value of the effective refractive index obtained from the phase analysis at the same frequency. We have summarized the values of $n_{\text {eff }}$ in Table I, and it is clear that the values obtained by the two different methods agree very well.

\section{CONCLUSIONS}

We have presented a detailed analysis of the propagation of $\mathrm{THz}$ SPPs through gratings of grooves structured in $\mathrm{Si}$. An
TABLE I. SPPs effective refractive index of gratings of 30 grooves structured on $p$-doped $\left(N \approx 10^{19} \mathrm{~cm}^{-3}\right)$ and $n$-doped $\mathrm{Si}\left(N \approx 10^{18} \mathrm{~cm}^{-3}\right)$. The lattice constant is $a=442 \mu \mathrm{m}$ and the groove depth is $d \simeq 100 \mu \mathrm{m}$. The effective refractive index is given at the frequency $\nu_{0}$ as obtained from the conical transmission of SPPs through the gratings $n_{\mathrm{eff}}^{\text {conical }}$ and from the phase analysis of the THz pulses transmitted at normal incidence $n_{\mathrm{eff}}^{\text {phase }}$.

\begin{tabular}{lccc}
\hline \hline & $\nu_{0}(\mathrm{THz})$ & $n_{\mathrm{eff}}^{\text {conical }}$ & $n_{\mathrm{eff}}^{\text {phase }}$ \\
\hline$p$-doped $\mathrm{Si}$ & $0.264 \pm 0.004$ & $1.05 \pm 0.01$ & $1.042 \pm 0.003$ \\
$n$-doped Si & $0.261 \pm 0.004$ & $1.09 \pm 0.01$ & $1.089 \pm 0.001$ \\
\hline \hline
\end{tabular}

increase of the groove depth leads to an asymmetric broadening of the stop gap and a shift of the central gap frequency to lower frequencies. The experimental results are in good agreement with the shift predicted by calculations. By changing the number of periods or grooves we have determined the decay lengths of SPPs in the Bragg gratings or the gratings scattering strength. The decay length is shorter for gratings in $n$-doped silicon compared to the ones in $p$-doped $\mathrm{Si}$ indicating the stronger scattering in the former gratings. We have also shown two methods for determining the effective refractive index of the gratings. The values calculated from the shift of the gap for conical propagation of SPPs and the values determined by the phase of the $\mathrm{THz}$ pulse are consistent with each other and close to one.

\section{ACKNOWLEDGMENTS}

The authors gratefully acknowledges financial support from the European Union through the projects Interaction (HPRN-CT-2002-00206) and Metamorphose (NMP3-CT2004-500252), and from the Deutsche Forschungsgemeinschaft. The work of J.G.R. was supported in part by the "Stichting voor Fundamenteel Onderzoek der Materie (FOM)," which is financially supported by the "Nederlandse Organisatie voor Wetenschappelijk Onderzoek (NWO)." The work of J.A.S.G. was supported in part by the Spanish Comunidad de Madrid (Grant S-0505/TIC-019) and MEC (Grant Nos. FIS2006-07894 and FIS2004-0108).

${ }^{1}$ H. Raether, Surface Plasmons on Smooth and Rough Surfaces and on Gratings (Springer, New York, 1988).

${ }^{2}$ J. Homola, S. Yee, and G. Gauglitz, Sens. Actuators B 54, 3 (1999).

${ }^{3}$ S. Bozhevolnyi and F. Pudonin, Phys. Rev. Lett. 78, 2823 (1997).

${ }^{4}$ T. Ebbesen, H. Lezec, H. Ghaemi, P. Thio, and T. Wolff, Nature (London) 391, 667 (1998).

${ }^{5}$ J. Krenn, B. Lamprecht, H. Ditlbacher, G. Schider, M. Salerno, A. Leitner, and F. Aussenegg, Europhys. Lett. 60, 663 (2002).

${ }^{6}$ W.L. Barnes, A. Dereux, and T. W. Ebbesen, Nature (London) 424, 824 (2003).

${ }^{7}$ S. I. Bozhevolnyi, V. S. Volkov, E. Devaux, and T. W. Ebbesen, Phys. Rev. Lett. 95, 046802 (2005).

${ }^{8}$ W. Barnes, T. Preist, S. Kitson, J. Sambles, N. Cotter, and D. Nash, Phys. Rev. B 51, 11164 (1995).

${ }^{9}$ S. C. Kitson, W. L. Barnes, and J. R. Sambles, Phys. Rev. Lett. 77, 2670 (1996).

${ }^{10}$ J. Yoon, G. Lee, S. Ho Song, C.-H. Oh, and P.-S. Kim, J. Appl. Phys. 94, 123 (2003).

${ }^{11}$ J.-C. Weeber, Y. Lacroute, A. Dereux, E. Devaux, T. Ebbesen, C. Girard, M. Ujué González, and A.-L. Baudrion, Phys. Rev. B 70, 235406 (2004).

${ }^{12}$ W. Barnes, S. Kitson, T. Preist, and J. Sambles, J. Opt. Soc. Am. A 14, 1654 (1997).

${ }^{13}$ I. Smolyaninov, W. Atia, and C. Davis, Phys. Rev. B 59, 2454 (1999).

${ }^{14}$ S. Bozhevolnyi, V. Volkov, K. Leosson, and A. Boltasseva, Appl. Phys. Lett. 79, 1076 (2001). 
${ }^{15}$ I. I. Smolyaninov, D. L. Mazzoni, and C C.. Davis, Phys. Rev. Lett. 77, 3877 (1996).

${ }^{16}$ J. G. Rivas, M. Kuttge, P. Haring Bolivar, H. Kurz, and J. Sánchez-Gil, Phys. Rev. Lett. 93, 256804 (2004).

${ }^{17}$ J. Krenn, H. Ditlbacher, G. Schider, A. Hohenau, A. Leitner, and F. Aussenegg, J. Microsc. 209, 167 (2002).

${ }^{18}$ D. L. Mills, Phys. Rev. B 15, 3097 (1977).

${ }^{19}$ V. N. Konopsky and E. V. Alieva, J. Mod. Opt. 48, 1597 (2001).

${ }^{20}$ W. Barnes, W. Preist, S. Kitson, and J. Sambles, Phys. Rev. B 54, 6227 (1996).

${ }^{21}$ S. J. Allen, Jr., D. C. Tsui, and R. A. Logan, Phys. Rev. Lett. 38, 980 (1977).

${ }^{22}$ D. Grischkowsky, S. Keiding, M. van Exter, and Ch. Fattinger, J. Opt. Soc. Am. B 7, 2006 (1990).

${ }^{23}$ J. A. Sánchez-Gil and J. G. Rivas, Phys. Rev. B 73, 205410 (2006).
${ }^{24}$ D. Grischkowsky, S. Keiding, M. van Exter, and Ch. Fattinger, J. Opt. Soc. Am. B 7, 2006 (1990).

${ }^{25}$ J. Saxler, J. G. Rivas, C. Janke, H. Pellemans, P. Haring Bolivar, and H. Kurz, Phys. Rev. B 69, 155427 (2004).

${ }^{26}$ J. G. Rivas, C. Schotsch, P. Haring Bolivar, and H. Kurz, Phys. Rev. B 68 , 201306 (2003).

${ }^{27}$ H. Hirori, K. Yamashita, M. Nagai, and K. Tanaka, Jpn. J. Appl. Phys., Part 2 43, L1287 (2004).

${ }^{28}$ E. Alieva, G. Beitel, L. Kuzik, A. Sigarev, and V. Yakolev, Appl. Spectrosc. 51, 584 (1997).

${ }^{29}$ J. A. Sánchez-Gil, Phys. Rev. B 53, 10317 (1996).

${ }^{30}$ J. A. Sánchez-Gil and A. Maradudin, Phys. Rev. B 60, 8359 (1999).

${ }^{31}$ J. G. Rivas, M. Kuttge, H. Kurz, P. Haring Bolívar, and J. A. Sánchez-Gil, Appl. Phys. Lett. 88, 082106 (2006).

${ }^{32}$ J. A. Sánchez-Gil and A. Maradudin, Opt. Express 12, 883 (2004). 